Journal of World-Historical Information

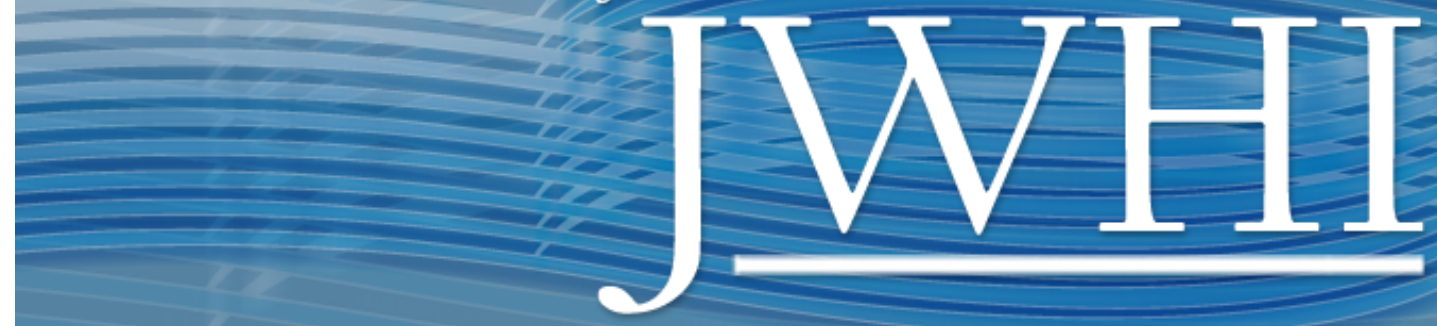

\title{
Cross-Disciplinary Theory \\ in Construction of a World-Historical Archive
}

\section{Abstract}

Copy and paste abstract article here. yet difficult goals to achieve in social science research. The nuanced differences among the domains, frameworks, assumptions, and methods of the various fields of study that comprise such research often hinder attempts to engage in interdisciplinary dialogue that is both meaningful and productive. We show that demography, economics, political science, and sociology are a few of the fields at the vanguard of the interdisciplinary frontier that emerged following the Second World War. In light of the challenges that these fields (along with the natural sciences) face in initiating and sustaining interdisciplinary dialogue, we aim to accomplish several tasks. First, we seek to describe the theoretical and epistemological linkages among the cores of these four social-science disciplines. Second, we explore systems theory as a potential foundation for interdisciplinary unity. Third, we extrapolate the implications of the systems approach to encompass the study of human populations from multiple disciplinary perspectives. In this vein, we also seek to characterize key features of human populations, parse their functions in various disciplinary contexts, and prospectively identify challenges in data interpretation and analysis that will likely emerge in practice. The ultimate goals of this study are to delineate a set of methodological standards with which to guide interdisciplinary inquiry in the social and natural sciences, and consider how we might implement these standards in the construction of a world-historical data archive.

Volume 1, No. 1 (2013) | ISSN 2169-0812 (online)

DOI 10.5195/jwhi.2013.3 | http://jwhi.pitt.edu 


\section{Patrick Manning and Sanjana Ravi}

\section{Cross-Disciplinary Theory in Construction of a World-Historical Archive}

\section{Introduction}

This is an eclectic overview of social-science theory and its interdisciplinary connections, with a practical objective: to clear the ground for constructing a large-scale historical data resource that ranges across social-science data with links to natural-science data in health and climate. The hope of finding empirical patterns at the global level - and of finding empirical linkages among various sorts of human experience over time-requires that analysts spell out theoretical linkages among social sciences. If we can't link social sciences explicitly into a larger web of theory about human behavior, we are left with a positivistic segregation of economy from society and culture as the best we can do in analysis. Such an approach, which still survives in parts of the academy, runs counter to the widespread intuitive sense that we live in a highly connected and interactive society. Our limited ability to express this connectedness in theoretical terms means that, in describing a past that precedes our contemporary intuition, we have only the analytical tools of segregated disciplines. World historians have been writing narratives that suggest a global interweaving of complex historical dynamics, but they have made little progress in formalizing their interpretations. ${ }^{1}$ For lack of clear statements on past links among the domains of economics, society, and politics, we underestimate the degree of past connectedness in human affairs. As a result, our comparisons of past and present overestimate the rate of change in social interaction. In turn, such miscalculation of current rates of globalization exaggerates and misdirects our ability to intervene with policy to address current crises and to project future changes. Fortunately, there have been some advances in exploring interdisciplinary connections in the social sciences. These advances have been achieved particularly at macro levels, for instance in historical world-systems analysis and in studies of contemporary globalization. In addition, many more cross-disciplinary insights have doubtless been developed but remain below the radar for lack of a sufficiently robust overall discourse on unifying the social sciences. ${ }^{2}$

We will encounter a vast thicket of problems in attempting to construct a comprehensive yet user-friendly world-historical data resource. So far, we are guided by two principles in decisions on which way to turn in structuring and analyzing data. First, we keep an eye focused firmly on macro levels, but aim to work principally from micro levels. The task at hand is that of constructing a world-historical data resource, which means working from relatively specific and localized levels of documentation to broader scales through processes of aggregation until we reach the global level. ${ }^{3}$ Second, we focus principally on the human factor in analysis-placing the human 
dimension (in all its complexity) before the dimensions of space, time, and the variety of topics. We assume that populations-human individuals and groups, as described in the field of demography-provide a starting point in our search for the links and common elements among the various social-science disciplines. From this standpoint we work at the micro level to establish the initial analytical links of demography to population-related variables in economics, sociology, and political science. In a briefer section that combines micro and macro levels, we have selected migration (a dynamic factor in demography) to indicate how we would analyze a relatively complete historical data resource.

We express our presentation in terms of systems. First, we consider the various real-world social domains of economic, social, and cultural life, and treat them as interacting sub-systems within an overarching historical system of human society. Second, we treat academic disciplines as symbolic systems for analyzing these social domains. Discussion within this essay takes place, on one level, through a comparative approach, in which we compare and contrast the various social and disciplinary sub-systems. On another level, we develop an interactive approach that traces threads and dynamics linking sub-systems, especially within the symbolic system modeling the real world. Of the two levels, we are most hopeful of achieving insights through the latter, interactive approach.

\section{Unifying Social Sciences: Previous Discussions}

The aftermath of World War II brought a resurgence of social science research accompanied by disciplinary reorganization and sub-specialization. The formation of UNESCO brought an impulse to global cooperation in social-science research. ${ }^{4}$ International organizations, congresses, and journals expanded substantially, along with research on large-scale issues intended to aid in postwar reconstruction and facilitate understanding of the world's sociopolitical landscape (Backhouse and Fontaine 2011). Some of the fields at the forefront of this academic transformation included psychology, sociology, anthropology, economics, and political science. Macrosociology developed in the form of Parsonian modernization theory; macroeconomics expanded through national income analysis. Subsequently, as quantitative techniques improved, individual-level analysis expanded with social surveys and microeconomic studies. These two directions of advance gave rise to observations on their differences: the "ecological fallacy" correctly identified the dangers of attributing macro-level correlations to individual behavior (Robinson 1950). In retrospect, however, it becomes clear that the trend was toward segregation of micro and macro analyses rather than productive connection through multi-scale investigation.

A look further back in time shows that this postwar expansion in research fit into a longer-term, cyclical pattern. Following the relatively specific analyses of the eighteenth century, Marx and Comte, each in his own way, proposed a grand mid-nineteenth-century synthesis of the social sciences. ${ }^{5}$ Later in the century the main developments in economics, sociology, and even politics focused on micro levels. As the twentieth century unfolded, larger-scale work emerged in sociology (Weber) and economics (Leontief and Keynes). Postwar work brought important theoretical and methodological advances on multiple scales, most notably with the development of systems analysis. One of the biggest practical advances was the extension of national income accounting to most countries: the United Nations and later the World Bank created and diffused comparative national statistics. Most social-science advances, however, took the form of developing additional silos within which new specializations grew.

Cross-disciplinary analysis was slow to develop. ${ }^{6}$ Although expanded use of statistical techniques provided some uniformity in vocabulary, for the most part the terminology of social scientists diverged from discipline to 
discipline and among specializations within disciplines. There arose intra-disciplinary discord over proper protocols, differing units of study, the seeming superiority or inferiority of certain sub-fields, and the validity of new data collection techniques. Numerous methodological barriers presented further challenges to interdisciplinary work. In response to the divergences and specificities of social-science analysis, some studies have sought to bridge the gaps. The methodological overview of King, Keohane, and Verba (1994) expanded a terminology emphasizing causal and descriptive inference, showing the parallels in reasoning throughout the social sciences. In work such as this, interdisciplinary analysis became a theoretical focus of social sciences in the 1980s and early 1990s. Then the interest in interdisciplinary study died down for some time, so that "Interdisciplinary Studies" survives institutionally as an undergraduate major rather than a research field. The Journal of Interdisciplinary History is one of few historical venues that emphasizes social-scientific analysis, but its publications tend to focus on small-scale, individual projects. In institutional terms, the International Studies Association and the Journal of World-Systems Analysis are two venues for practical cross-disciplinary analysis; so also is the American Association of Geographers.

Both the need and the resources for interdisciplinary analysis are again advancing, as indicated by a recent methodological synthesis (Gerring 2012) and a history of overlapping social sciences (Backhouse and Fontaine 2011). Successive developments in computer technology brought better handling of data by permitting large-scale handling of tabular data - for simple storage, for arithmetic calculations as in demography and through statistical analysis. Still, the fields ran parallel to one another. The effort to create a global historical data resource thus creates pressure for more linkage among fields. Still, without a cohesive strategy on hand to integrate such findings within the larger context of the human condition, the potential utility of a world-historical data resource is limited at best.

\section{Real-world Social Domains vs. Symbolic Systems of Disciplinary Analysis}

Our ultimate focus is on the real-world domain of global human society during the past 500 years. We seek to develop global and regional time series for major variables on demography, economy, society, politics, climate, and health. Yet to clarify our understanding of historical human society we must proceed by distinguishing between the real world itself and the symbolic world of our disciplines and theories. This distinction, while widely understood, is not often formalized. We formalize it here in the hope that it will clarify problems we expect to encounter in laying the groundwork for a broad system of social archiving and analysis. A given real-world domain has its inherent dynamics and processes. ${ }^{7}$ As we will see, the disciplines, however parallel they are to the real-world domain, are different in the additional boundaries that exist in symbolic systems and in the processes of change or evolution in symbolic systems as compared to those in real-world domains. In effect, they are parallel universes. Let us adopt the term "Social Universe 1" to describe the real world of human society, and the term "Social Universe 2" to describe the parallel but symbolic world of social-science disciplines

We begin with a concise statement of a systems-based understanding of Social Universe 1, the real-world domain of human society over the past several centuries. We can envision world-historical society as governed by an underlying, systematic set of processes and that has developed and interacted to reproduce itself, change, and grow. To understand its components, we can imagine domains and sub-systems defined variously by region, time, topic, populations, perspective, and level of aggregation. The real-world economic domain, for instance, includes an interconnected set of relations in production, exchange, and distribution of goods and services-mediated nowadays by monetary and financial practices. The economic system has its edges, its input, its processes, and its output. But 
one must also account for the various levels at which this system operates, from the individual decisions people make on a daily basis to national and global economic patterns. Similarly, we can imagine that social, political, and cultural systems of various times and places fit together to form a grand, real-world, historical system.

We model this real-world system with symbolic systems analysis: this is Social Universe 2. When we construct and modify models of human society, we act in the field of human ideas. Within this great and subjective field of all ideas, we narrow our attention to the arena of interpreting historical human society. We imagine the real world as a system, and then formalize it as a symbolic system of data and interpretation, organized by theory, and subdivided by discipline. Drawing back to view the full range of Social Universe 2, we can see it as a complex, multidimensional space, representing human society: it encompasses a grid of topical fields and social groupings spread along dimensions of space and time. ${ }^{8}$ We have the option to study social and historical dynamics within localized, sub-disciplinary regions of this analytical space; we can also explore links among subfields and the functioning of larger realms.

In this essay we consider the social domains of population, economy, society, and politics. Alongside each of these social domains we set the specific social-science disciplines which we have chosen as the real focus of our study: demography, economics, sociology, and political science. Each of these disciplines is a symbolic system sustained by artisanal practices and tools such as data collection and linear regression. Each discipline is a system of thought with its characteristic dynamics; it has its input, its system of reproduction and feedback, and its output. These processes and interactions take place at various levels from the localized and specific to the broad and general: we now accept that there are global patterns in population, economy, society, and even politics. That is, we assume that real-world social domains exhibit overall systemic behavior, at multiple levels, which are susceptible to analysis through systemic theories.

\section{A Tentative Terminology and Framework for Disciplinary Linkage}

Here we offer a description of our terminology; we offer further terminological detail in Appendix 1. We have already introduced the terms of Social Universe 1 and 2, along with disciplines. We propose that, within Social Universe 2, each discipline has a basic set of variables, organized into four principal dimensions: time, space, topics, and populations. Each discipline also traces "transitions" and describes "institutions". The interactions among disciplines, which generate the larger social system, consist of "inputs" to each system from others plus "outputs" from each system to others; these bring about "feedback" in sum.

"Data" are observations based on Social Universe 1, especially as recorded in historical documentation; the object of our project is to enter them into Social Universe 2. In addition to data based on direct observation, we can hope to fill in missing data through reliance on the interplay of existing data and the disciplines.

"Levels," "dimensions," and "scales." The disciplines work at multiple levels of aggregation. We use the term "level" to refer to different strata along each dimension. We use "dimension" to refer to four characteristics of disciplines and disciplinary information, largely orthogonal to each other: time, space, populations or social groups studied through the discipline, and topics of analysis within the discipline. We consider varying levels for each dimension: the lowest level is generally a single day (in time), the extent of a single household (in space), the individual person (in population), and a single item of a given type within topics. Levels of spatial and temporal dimensions, along with varying extents of population, are ordinal (that is, we may rank them in scale from small to 
large); topics within social-topical domain are categorical (difficult to rank). We hope to reserve the term "scale" to refer generally to both levels and dimensions. That is, "large scale" may refer to aggregation of some but not all aspects of a system: the geographic area, the time encompassed, the range of topics encompassed, or the overall collection of elements in the system. ${ }^{9}$ The term "scale," while useful, is nevertheless somewhat ambiguous.

"Aggregation" and "organization." We pay particular attention to aggregation in that much of the work in constructing a world-historical data resource will consist in aggregating localized data into larger-scale totals. At each level the data may be described in aggregated terms (not showing the breakdown) or disaggregated terms (showing the breakdown). For instance, figures on national income could reveal simply the aggregate total or give breakdowns by province or industry. It is a priority for us to retain the localized data as we aggregate them, to permit analysis of interactions among local and global levels. While aggregation is characterized by simple joining or grouping, "organization" in a systems context involves the meaningful arrangement of systemic components, lending the system in question a teleological function. To discuss multiple levels in these phenomena, one may introduce the terminology of a "spectrum of aggregation" or a "spectrum of organization."

"Higher order" and "lower order" systems or scales. These allow for comparing scales of a system, where "higher" and "lower" refer broadly to the scope along various dimensions and the number of elements in the system at each level: hence, the difference between microeconomics and macroeconomics or between local government and imperial government. "Emergent properties" are systemic properties arising at a higher-order level from interactions at a lower-order level; there may also be systemic properties arising at a lower-order level from the influence of higher-order systemic behavior.

"Complexity." We have explored the literature on complex systems, to see whether it introduces additional essential elements to our analysis (Casti 1986, Flood and Carson 1993, Harvey and Reed 1996, Liu et al. 2007, Tsoukas and Hatch 2001). We recognize the widely varying range of complexity in systems, but so far we have found no general thresholds in social systems requiring us to focus explicitly on levels of complexity. As a result we assign no specific role for "complexity," on the assumption that everything in social life is more or less complex.

Having specified our terminology, we now rely on it for summarizing our framework. Considering the full range of systems and potential cross-system links within Social Universe 2, we must ask: which processes and links do we model? Further, to build a global historical data resource, we must ask: how do we link the models to data? We repeat our observation that linking the varying scales has been relatively neglected within disciplines and in interdisciplinary analysis. We have the impression that, for each of the four disciplines under study here, the analysis began with concerns at multiple levels - for instance, with the process of government overall, but with attention to the dynamics in a single court. Or with concern for social conflict overall, but with attention to families. One indication of the growing attention to scale is the emergence of "meso-level" theories, for instance in migration theory.$^{10}$ Henceforth it appears that we will work with individual, micro, meso, and macro scales - although one may expect that these are not entirely sufficient, and that adding "meso" into the mix will not be definitive.

The scope of each discipline is large indeed. Theorists have selected-out of the full range of possibilities - the processes, social arenas, levels of aggregation, time frames and spatial ranges with which to specify relationships and dynamics that they find to be important. It is evident, however, that the theories are not comprehensive, in that they address only a portion of the relationships within their disciplinary scope. Thus, as new theories are developed in social sciences, some of the new work is to replace and improve on old theories, while other new work is to theorize issues that have not previously been analyzed. ${ }^{11}$ 


\section{Symbolic systems: properties and dilemmas}

Now we turn to the formal treatment of disciplinary systems, including their sub-systems and interactions. While each disciplinary theory reflects an effort to model real-world dynamics, symbolic-theoretical systems cannot be equivalent to real-world systems. To illustrate, here are several points on the properties of disciplinary systems, along with the advantages and dilemmas they bring to analysis.

Symbolic systems are bounded, while real-world systems shade into each other temporally and in other dimensions. The analytic definition of system boundaries is always arbitrary and provisional, set as a convention by the analyst. In the real world, in contrast, the analyst's symbolic vision of discrete systems is violated at every turn by the interplay and overlap of real-world systems from the molecular level to galaxies and beyond. That is, Social Universe 1 is not really bounded in the way we assume within Social Universe $2 .{ }^{12}$ This is perhaps the biggest difference between real-world systems and symbolic systems. For instance, the analytical specification of a system always requires the setting of initial conditions for the moment when the analysis begins; in the real world, time does not start or stop, so that the conditions and endowments at each moment are inherited from the moment before rather than given separately. The dilemma of boundaries in analytical systems is that, while they permit simplification of analysis, they may neglect important cross-boundary effects.

Disciplinary systems function with distinct but overlapping topics and scales. For each disciplinary system, we can define disciplinary sub-systems according to varying criteria by topic and scale. Thus economics distinguishes money and banking from firm behavior, microeconomics from macroeconomics, and specialists on European and Japanese economies. It is important to note the parallels and distinctions among the dimensions of time, space, population, and topic. Each can be treated at varying levels of aggregation. The difference is that the amount of time, the area of space, and the extent of populations considered can be ranked in ordinal terms, while the differences among topics cannot generally be ranked in ordinal terms.

The elements of disciplinary systems exist in relation to one another; none serves as an essential building block. For the physical world, one could attempt to argue that atomic particles or chemical species are the elemental forms from which all else is constructed. For the world of human society, however, even the most basic elements (individuals, households, families) are immensely complex. As a result, elements and subsystems at all levels may influence each other. By the same token, there exists no privileged vantage point which can give definitive interpretation to the system: theories and interpretations are partial, and depend on the range of perspectives from which they are constructed..$^{13}$

Linkages among disciplinary systems require additional theorization. The various disciplines, while commonly developed in imitation of each other (so that the notion of equilibrium recurs commonly), differ substantially. Thus, even while sociology, political science, and economics share numerous variables, it appears necessary to construct new theoretical statements in order to link them to each other. By the same token, for the various and widely differentiated subfields within a given discipline, additional theoretical statements need to be constructed to explain the interactions among the domains of the subfields. A systems approach draws attention to the benefits of exploring links among these sub-systems, but one faces the difficulty of resolving conflicts in local terminology. 
Disciplinary systems need to model bottom-up adaptations that create “emergent properties.” Emergent properties are "qualities not included in, and generally not predictable from, knowledge of the qualities of the systems in which they arise" (Wilden 170; see also Appendix 2). The systemic notion of emergent properties can be very helpful in understanding historical change, in that it indicates that the rise of states or long-distance trade came not simply through the agency of individuals but through adaptation of a system to problems undermining its functioning. This concept relies on notions of scale or levels of aggregation, in that the emergent property of a system is defined as taking form at a higher order of complexity than the phenomena from which it emerges; it shows the functioning and change of adaptive systems. A dilemma arises, however, in the contrast between modeling emergent properties in real-world systems and emergent properties of theories themselves.

Disciplinary systems need also to model top-down systemic adaptation. At the same time, it is surely illogical to limit the study of social-system linkages to the emergence of new and broader structures out of parochial elements. Higher-order elements of a system can bring change at more localized levels: states, for instance, have not uncommonly brought about changes in family structure. ${ }^{14}$ As a result, there is need for theorization-parallel to that on emergent properties - of the adaptations in localized systems in response to the pressures from higher-order systems.

Symbolic systems help to explain real-world social evolution. More generally within this same systems framework, we can image that Social Universe 1 undergoes "social evolution" as a particular sort of systemic change (including the development of emergent properties such as empires and global markets), and that we can try to theorize such evolution within social-science disciplines. The term "social evolution" is clearly metaphoric, comparing human social change with other types of generalized change. For instance, we can consider the slow change in the inorganic, physical world, as told in the narratives of Big History: the early formation of atomic particles, the emergence of chemical elements, then of stars and of solar systems, and the successive changes in the geology of the earth.(Christian 2009) In contrast to this inorganic story we can consider the later but more rapid rise of life forms through organic evolution - in this case a very specific mechanism of evolution centered on DNA, which brought a succession of species leading up to our own. Thirdly, one can speak of human social evolution. In this case, consciousness plays an unprecedented role in the changes in human technology and social organization. While the mechanism for change is less clearly defined than for DNA, the rate of social evolution is certainly more rapid than that of biological evolution. In systemic terms, all of these types of evolution can be treated as situations in which systems develop increasing complexity. ${ }^{15}$ The dilemma in interpreting social evolution, however, is that varying social processes have quite different rates of change, so that it is difficult to distinguish short-term fluctuation from longer-term evolution.

The dynamics of change in social-science theory are only partly understood. We assume that Social Universe 1 refers to the entire system of human society, including its fluctuations and evolution. Is Social Universe 2, our model of historical society, fully comparable to the real world? Manifestly not: at present it falls far short of effectively modeling Social Universe 1. We can imagine at least three processes by which Social Universe 2 may become a steadily better model of Social Universe 1: revelation, agency, and evolution. In the first case, we can imagine that a pre-existing, comprehensive theoretical system exists implicitly in some adjoining dimension, waiting to be revealed with the details of its analysis of global society: in that case its direction is inevitable. In the second case, we can imagine that world-historical social-science theory must be constructed and assembled by conscious agency and initiative: in that case its direction is fully contingent. In the third case, we can assume that broad socialscience theory will evolve by some systemic and unconscious mechanism in association with human agency, in a development somewhat parallel to the evolution of historical society itself. These alternative paths of disciplinary 
change await further study. In any case, we can be certain that present-day social-science theory is inadequate to the task of analyzing the real-world global-historical system. In sum, a systems approach is likely to be of assistance in developing or discovering interdisciplinary theory. One dilemma is likely to arise from the tendency of individual theorists to exaggerate the role of their individual agency in determining the direction of theory.

Disciplinary narratives. In practice, each discipline sustains a narrative of its development, tracing the succession of analytical foci and theoretical formulations of its leading practitioners. ${ }^{16}$ These narratives make the case that each discipline has expanded in scope and sophistication with time, but it is not clear whether such chronicles of disciplinary change should be seen as reflecting a sort of evolution of Social Universe 2. We may ask whether there is an overall mechanism or process for development of disciplinary knowledge. The post-World War renaissance of the social sciences, for example, demonstrates the role of sociopolitical factors in shaping the current foci and scopes of the disciplines: social movements, decolonization, and the welfare state brought major advances in social-science analysis. Narratives such as this actually serve as valuable descriptive tools, although some scholars might question the subjectivity - and consequently, the reliability - of narratives in analyzing complicated systems. Narratives allow for alternatives to triumphalist tales of disciplinary advance; they hold on to complexities and incongruities and feed them back into later analysis. Because they present us with a method for metaphorically capturing the nuances of micro-level phenomena, narratives may in fact function as important theoretical linkages between occurrences in real-world systems and the aforementioned symbolic systems of disciplinary analysis. Equally clear is that the authors of the present study hope that an organized effort to assemble a wide range of current social-science theory and practice will advance substantially the theory of world-historical change and interaction.

Our problem is how to advance theoretical statements that convey our understanding of the interplay among the social sub-systems in human society. As theorists, we seek to expand and clarify the parallels between each scholarly discipline and the equivalent domain of real-world conditions. At the same time, we seek to locate and develop links among disciplines, thereby locating previously neglected links among the various real-world domains. This mix of parallels and links among disciplinary systems is key to the game of interdisciplinary analysis.

Data Integration and analysis. Our primary aim in this article is to establish conceptual and theoretical foundations for the eventual construction of a world-historical dataset spanning multiple disciplinary domains. However, the prospect of tackling the latter endeavor raises pressing questions about data management, integration, and analysis, with respect to our theory of symbolic and real-world systems. Addressing these issues is critical to achieving our goals of effectively linking the two distinct, but related, social universes and of clarifying links throughout Social Universe 2. We recognize that the qualitative and quantitative data entering our repository, gathered from numerous, possibly disparate sources, are themselves artifacts of the real world, Social Universe 1. In order to model real-world phenomena using these data with accuracy, however, we must synthesize them within the theoretical confines of Social Universe 2; that is, integrate diverse data in such a way that they are comparable to each other and may also be used to develop cohesive theories about the mechanics of Social Universe 1. Given the likely difficulties associated with synthesizing possibly incongruent data, we propose to employ a meta-analytical approach (i.e. combining the results of multiple, multi-disciplinary studies) to evaluate and integrate incoming data, which in turn will allow us to draw new, methodologically sound conclusions about real-world phenomena. Because our world-historical archive will consist of both quantitative and qualitative data, we will have to apply different methods as we move forward with this approach. Within political science, for instance, Thacker provides examples of several quantitative strategies for analyzing voting data pulled from multiple studies: simple counting, clustering, and pooling. ${ }^{17}$ Each of these allows researchers to study different facets of a real-world phenomenon on a common 
scale, and link disparate data via statistical analysis. In another example, there exists a plethora of data on climate, much of which describes temperature and weather conditions on a day-to-day basis. Most disease incidence data, by contrast, is typically collected on a monthly or yearly basis. Correlating climate patterns with the emergence of disease, therefore, is problematic due to the differing temporal scales on which these data exist. The strategies described above promise to work well for combining numerical data, provided the data in question is standardized to the same temporal, spatial, and/or demographic scale(s) of analysis. Integrating numerical information with qualitative data, on the other hand, also proves be challenging. Parsing qualitative data - such as anecdotes, narratives, or art, for example - requires researchers to operate on scales of analysis that may not be wholly compatible with quantitative scales. Nevertheless, qualitative data may prove to be useful in terms of structuring the interpretive process; that is, using them as a means of deciphering the meaning of numerical information.

\section{Applying the framework to four disciplines}

In this section we seek to convey an impression of how to link disciplines in a large data resource by describing and linking key characteristics of four social-science disciplines. While it would ultimately be beneficial to develop equivalent descriptions for each and every social science, the introductory nature of this essay makes it appropriate to limit our descriptions to four disciplines, taking demography as the most basic. ${ }^{18}$ For each discipline we begin with a concise and schematic statement of its character: purpose, scope, main variables, dimensions (space, time, topic, population - at varying social scales indicated above); plus key transitions, processes, and institutions for each discipline, and the main inputs and outputs by disciplinary systems is connected to others.

Next, we consider the multi-level links within each discipline. Fitting the variables together will require attention to aggregation and disaggregation, and attention to temporal movement or migration (across space or topic) within periods and between periods. Movement across time is not reversible; movements across space, social roles and topics are reversible. Discussion within each discipline will depend at once on elements central to the disciplinary theory and on elements included in practice among historical data.

The most important step for us the next one, in which we trace cross-disciplinary links for each discipline. Of particular importance are links involving demographic variables and data. That is, our hypothesis is that human participation is the most essential element of social sciences, and that demographic variables are the closest variables in the social sciences to that human essence. The accompanying figure portrays four disciplines, each containing internal linkages among its basic and higher-order elements (light arrows). The dark arrows show the inputs and outputs linking demography with each of the other disciplines - these are the links we identify in the text. To be complete, the analysis needs to consider both theoretical variables and practical categories of data. In addition, there are also interdisciplinary links among economics, sociology, and political science, but the figure shows them as simple dotted lines and the text does not include them because we have not yet attempted to specify them. 


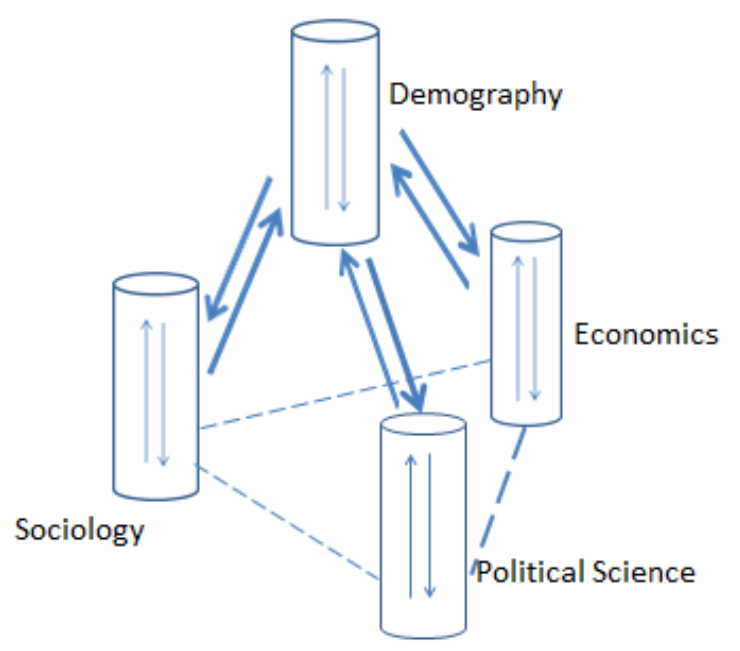

In the concluding discussion for each discipline, we consider higher-order feedback among disciplines. At this early stage in our work we are not able to offer precision in these discussions, but we seek to initiate a sustained discussion of the higher-order relationships among disciplines will ultimately be important for our construction of a world-historical data resource.

\section{Demography.}

Basic character of the discipline:

Purpose: study of human birth, life and death

Variables: age, sex, birth, status, death

\section{Dimensions:}

- Spatial domain: individual, local, national

- Temporal domain: annual, 5-year periods, generations, life cycle

- Topical domain: age, sex, measures of social status

- Social-scale domain: individual, micro population, aggregate population

Transitions and processes: birth and death (as events and rates), social transitions (migration, marriage, social mobility)

Institutions: biological family

System inputs: births (plus numerous biological, social, and psychological inputs)

System outputs: deaths 
Multi-level links within the discipline. Demography begins with individual-level data, but also pays close attention to family, locality, and society, and especially to total populations within demographic units. Secondarily, demography focuses on geographic migration in and out of populations under study (by age and sex), with accompanying death rates. Demographic rates are usually calculated on an annual basis; censuses and population surveys are usually calculated in five-year age groups.

Cross-disciplinary links for demography with economics, sociology, and political science. For all variables including references to persons, social sciences should pay attention to the basic demographic variables of age, sex, and rates of birth and death. Demography can consider sub-populations (by social category) and migrations across social status (by economic, social, political, and other groups). To document these sub-populations and status migrations, demography must respond to the needs of adjoining disciplines, breaking down overall populations into the relevant sub-populations. Shifts among the most basic demographic categories, via birth and death, are not generally voluntary, but marriage and migration are relatively voluntary. Shifts among categories of religion, social class, political party, employment status are theorized according to variables within each field.

Higher-order links for demography. For other disciplines, populations are broken down by many types of status, social function, or social activity. Time frames may need to be shortened to as little as one day to encompass major social phenomena. Spatial locations of populations change with time.

\section{Economics.}

\section{Basic character of the discipline:}

Purpose: study of production, exchange, consumption [trade, money, employment, production]

Variables: prices, quantities, labor force (by age and sex), occupation, income, wealth, transportation; Macro pop, consumption, money, industry, input-output (per capita and aggregate)

\section{Dimensions:}

- Spatial domain: individual, local, national, international

- Temporal domain: quarterly, annual, decennial, 30-year, really long run

- Topical domain: prices, quantities, labor force, firms, households, incomes, wealth, resources, markets, money, employment status, profitability

- Social-scale domain: individual, household, firm, regional, national

Transitions and processes: growth, cycles

Institutions: firm, household, bank, government, labor and employer associations

System inputs: labor from population, funding from politics, administration from sociology.

System outputs: sustenance to population, revenue to politics, status goods to sociology.

Multi-level links within the discipline. While economic theory focuses on firms and households, economic statistics focus on regional markets, listing quantities of goods produced and sold, quantities of inputs (including labor), prices, quantities of goods transported from one market to another (presumed equal to quantities sold in the receiving market). To include economies from the most basic to the most elaborate, it is 
necessary to take explicit account of the productive sector, the consumer sector, the exchange sector, a sector for money and finance, and the government sector. An input-output approach to the economy distinguishes inputs of labor, raw materials, and intermediate goods in the production of final goods and services. Attention to social institutions and development of skills draws attention to human capital (in addition to physical and financial capital). Government provides demand for goods and services, supports costs of the bureaucracy and the military, and conducts regulation of the economy. Labor forces are described by occupation within a regional market. Money supplies can be estimated by regional market; flows of money among markets can be noted. Wealth is calculated on an individual basis for holders of significant wealth; it is commonly neglected for the rest. Other institutions of interest include regulators, banks, markets, productive firms, and transportation and other service firms.

Cross-disciplinary links for economics with demography. Population figures are in the numerator of economic variables addressing the labor force and the number of consumers. Populations are in the denominator of economic variables for productivity and for per capita figures for land, capital, income, wealth. For figures such as per-capita income, wealth, and consumption, when based on aggregate population, risk bias when they fail to account for shifts in population composition.

Higher-order links for economics. Human populations might be thought of as complex systems of decision-making operating on a positive feedback loop. That is, micro-level decisions result in the emergence of macro-level phenomena, which in turn inform future micro-level decision-making processes. A common pattern within economic activity, then, is optimization: the state in which the system operates with the greatest efficiency, maximizing resource usage and minimizing waste. This symbolic description of economics as a subsystem, however, is somewhat incongruous with real-world economics in that decision-making does not take place in a vacuum consisting solely of economic phenomena. Rationality, for example, is a phenomenon that arguably overlaps with the disciplinary domain of psychology, and is a powerful shaping force within the human decision-making process. Similarly, one must also account for the influence of social institutions (i.e. governmental regulation of fiscal policies) on the individual decisions that drive global economic patterns.

\section{Sociology.}

\section{Basic character of the discipline: ${ }^{19}$}

Purpose: study of relations among and within communities and social institutions [education, literacy, religious groups]

Variables: family, gender, religion, ethnicity, race, rural/urban, occupations, residence, education

\section{Dimensions:}

- Temporal domain: annual, generational (30-year) for community change; centuries for social change

- Spatial domain: household, local, rural or urban settings, national, civilizational

- Topical domain: family, community, ethnicity, religion, race, occupation, residence, cities, industry, health, education, status groups (free, slave, etc.), wealth, identity, legal status

- Social-scale domain: individual, family, community, society, civilization 
Transitions and processes: demographic transition, industrialization, modernity, social movements, social mobility

Institutions: social family, voluntary associations, community

System inputs: people to fill social roles from demography, socially significant goods from economics, support for certain social roles from politics.

System outputs: conditions for reproduction to demography, demand for socially significant goods to economics, constituencies to politics.

Multi-level links within the discipline. Descriptions are by community and community change; by nation and national change. Social institutions: families, community organizations (including diaspora), labor organizations, religious organizations, age and gender organizations, residential organizations.

Cross-disciplinary links for sociology with demography. Sociology identifies many sub-populations, by gender and age (in common with demography), but also by race, ethnicity, religion, race, education, legal status. These social groups are defined and studied at levels from family to community, society, and civilization. In addition, these specific populations are often reported on a per-capita basis in relation to total populations.

Higher-order links. Because variables such as culture, ethnicity, leadership, and other forms of social relations often serve as the factors that designate a society as a cohesive whole, it is difficult in practice - as is the case with economics - to pursue sociology in isolation from other forms of disciplinary analysis. Sociological inquiry thus represents an aggregated approach to understanding human population dynamics, albeit one that is highly dependent on the nature of interactions between lesser-aggregated phenomena.

\section{Political Science.}

\section{Basic character of the discipline: ${ }^{20}$}

Purpose: study of governance and public policy in states, to resolve differences among constituencies [comparative, international relations, public administration, public opinion].

Variables: local government, voters by society, legislation, parties, citizens, political units, military, regulation

\section{Dimensions:}

- Spatial domain: local, polity

- Temporal domain: years for elections and constituencies; generations for policies; up to two or three centuries for succession of governing regimes.

○ Topical domain: law, legislation, regimes, citizens, subjects, aliens, voters, philosophies, democracy, dictatorship, diplomacy, interest groups, constituents, interest groups, individuals

○ Social-scale domain: individual, micro, macro

Transitions and processes: regime change, war, regulation, alliances

Institutions: state, bureaucracy, military 
System inputs: population to govern from demography, revenue from economics, constituent groups from sociology.

System outputs: political direction to demography, regulations to economics, regulations to sociology.

Multi-level links within the discipline. The functions and topics of governance include identification of the constituents at any level (citizens, subjects, aliens, etc.), the legislation and regulation carried out through governance, the enforcement of laws and regulations, plus diplomacy and war with other political units. In addition, governments undergo patterns of regime change - rise and fall, constitutional changes, along with shifts in dynasties or office holders. Political science defines individual constituents but reports on them by constituency. Constituents obey or disobey the laws set by hegemonic authority; constituents may have formal or informal means of participation in creating or responding to legislation. Governing bodies exist at local, provincial, and central governmental levels. Interest groups participate at all levels. Other topics in political science include central authority, its constitution, the breadth of participation in its decisions; the effects and effectiveness of legislation and regulation; and the rise and fall of new governments and new governing regimes.

Cross-disciplinary links of political science with demography. Populations of constituents, citizens, subjects are defined by various political units. Since political science analyzes the government as well as the governed, it carries out smaller-scale (though perhaps higher-order) analysis of legislative bodies, court officials, bureaucrats, military officers and so forth, accounting for their numbers and their demographic characteristics.

Higher-order links. Like sociology, political science also represents an aggregated approach to studying populations that is nevertheless dependent upon disaggregated phenomena such as individual party affiliations or involvement in the political process. As a result, issues of scales and levels are particularly significant to the accuracy and relevance of political science studies as they pertain to the study of human populations. Gibson, et al. (2000), for example, discuss the problems associated with extrapolating micro-level findings to explain macro-level phenomena; conversely, one cannot consider majority-rule decisions representative of individual citizen preferences. Such decisions, however, are often the driving forces behind the formulation of policies and laws which in turn inform human decision-making and behavior.

\section{Migration: A look at later stages of archive construction}

We turn now to a brief exploration of higher-order disciplinary interactions of the sort that could be analyzed for elements of a relatively complete world-historical data-resource. This peek at later stages of analysis, intended to help guide early-stage decisions, focuses on the social role of migration. The process of migration is found in human populations across the globe, in various social milieus. Unlike the migratory behavior of other animals such as birds and fish, which is driven predominantly by biological factors, human migration is underpinned by strong social and institutional influences. Though much attention has been granted to the migratory act itself - the movement of individuals, populations, or communities from one location to another, over time - we now shift our focus from global patterns of human relocation to the microscopic phenomena (which span multiple disciplinary sub-systems) associated with migration. In our analysis, let us consider a few of these phenomena as micro-level variables within a complex human population. In this context, the term "migration" encompasses not only the 
relocation patterns of systemic components - people - but also the transition of said components among various social, political, and economic states. Migrants to a new country, for example, experience a shift in their political status (from citizens of their homeland to aliens in a different political state) and their social status (from being natives of one country to becoming foreigners in another). Similarly, a potential change in economic status (i.e. an increase in wealth or securing employment) is often a powerful motivating factor behind migrant behavior. We hypothesize, in turn, that such micro-level, sub-system-specific transitions as these set the stage for the macro-level patterns of migration typically handled in demography, as well as for other aggregative economic, social, and political phenomena. It is also crucial to note that the spatial and temporal scales on which these migrationassociated transitions occur do not always correlate neatly with the scales of the macro-level patterns.

Given these proposed transitions occurring in the various sub-systems in human populations in Social Universe 1, let us examine the process of migration using both Paulson's theory of self-organization and the framework of analysis established earlier in this study. In this manner, we hope to elucidate the parallel systemic mechanisms at play in Social Universe 2 (Paulson 1991). In a rudimentary sense, the movement of people represents spatial disaggregation and re-aggregation within populations. When we analyze this phenomenon in Social Universe 1 , however, we find that migrants do not simply conform to the dynamics of the populations they join. Instead, by actively introducing novel information to their new environments - in the form of customs, knowledge, skills, beliefs, and practices - they serve as active shapers of system dynamics. Therefore, the migratory act itself - the mechanism behind population disaggregation and re-aggregation - is not the sole factor responsible for macro- and micro-level migratory patterns observed in Social Universe 1 over time.

How, then, might we model these patterns in Social Universe 2? If we consider distinct human populations as unique sub-systems capable of interacting with each other, then the act of migration and its associated social, economic, political, and demographic transitions represent a transfer of systemic inputs. As discussed above, however, this transfer is not an entirely neat one - migrants introduce a variety of novel variables and information to their new environs. This phenomenon often results in significant overall changes in system dynamics. Paulson (1991) labels such new, non-contextualized, or unorganized information as "noise," or that which is extraneous at a given level of analysis. Despite the seeming unimportance of noise at a particular level, however, Paulson nevertheless maintains that noise is often an important indicator of other levels of systemic interactions. In terms of migration in Social Universe 2, such noise may in fact facilitate the emergence of new, unforeseen systemic properties, which in turn allows for the system to evolve across its associated symbolic domains - changes in economic activity, the establishment of new social and political institutions, and the formation of new social groups which are conducive to demographic analysis. In this way, symbolically modeling a process (migration) occurring in a defined system (human populations) over time and space provides insight into the mechanisms by which a system might evolve, while also underscoring the need for more sophisticated tools capable of parsing the inevitably more complex dynamics of said system.

By tracking the flow of information between and across disciplinary domains in Social Universe 2, we are able to draw conclusions about the relationships between these domains and their significance in the context of building a world-historical data resource comprised of information from Social Universe 1. When studying migratory patterns, for example, we see that individuals are capable of moving from one population (a sub-system) to another despite the fact that our systems of interest may possess distinct symbolic and real-world boundaries. These systems are still able to synthesize diverse inputs across numerous scales and levels. A migration data resource, therefore, would also benefit from possessing adequate data (spanning different disciplines) at varying levels of macro- and micro-analysis. Secondly, we find that migratory dynamics vary between these macro- and 
micro-levels of analysis; that is, patterns of migration observed globally may not necessarily be representative of the micro-level phenomena interacting with global patterns. Similarly, the dynamics we analyze in our symbolic framework, Social Universe 2, likely differ from the real-world migratory patterns we observe in Social Universe 1. Bridging this gap relies on our ability to develop analytical tools or identify proxies that accurately measure the changes stemming from migratory activity. Finally, we see that although global migratory dynamics differ from their micro-level counterparts, they nonetheless influence the future course of micro-level phenomena, often through feedback loops. Therefore, we might also consider the utility of a migration dataset in terms of our ability to use it to identify such patterns retrospectively, and hypothesize prospectively about future systemic phenomena.

\section{Conclusions}

To frame and conceptualize our task of creating a world-historical data resource, we have found it helpful to think of two universes of social interaction. First, we chose to think of historical human society as a great field of systemic interaction, propagating itself over time through processes taking place in overlapping social systems. Second, we imagined a universe of disciplinary analysis of historical human society, parallel to the real world. This formulation made clear the parallels in the disciplinary and real-world universes but also their differences. The framework helped to underscore the multiple dimensions of every discipline (we labeled them as time, space, topic, and population) and also the multiple and interacting levels of analysis within each discipline. At the same time, comparison of the two universes showed that the symbolic universe of disciplinary analysis, so far as we know, has many blank spaces where theories and linkages have not yet arisen as descriptions of the real world. Thus, as new theories are developed in social sciences, some of the new work is to replace and improve on old theories, while other new work is to theorize issues that have not previously been analyzed. ${ }^{21}$ Considering the full range of systems and potential cross-system links within Social Universe 2, it is necessary to reconsider: which processes and links do we model? Further, to build a global historical data resource, we must ask: how do we link the models to data?

Our formulation draws attention to two levels of complexity in disciplinary analysis. For each discipline we have noted the great internal variety in times, spaces, topics, and populations, and in the scales at which these are addressed. Disciplinary analysis, developing maps of disciplinary structure and interaction, including the complementarity and tensions of theories within a given discipline, is thus a significant part of our overall task. A related but distinct and larger-scale task is that of interdisciplinary analysis, the linkage of two or more aggregations of spaces, times, populations, and topics. This latter step is the one that will permit advances in our understanding of interactions among the various elements of human society through creation of a broad historical data resource.

One might phrase this research in terms of emergent properties. That is, one might say that, by calling for the formalization and linkage of disciplinary theories, with attention to the interplay of scales within each discipline, we are trying to provoke the rise of an overarching, interdisciplinary, social-science theory as an emergent property of academic discourse. In practical terms, such an emergent academic discourse on global society would facilitate debate on human society at the global level, through exploration of various models of the overall human social system and its sub-systems. We welcome such debate, but also hope for a degree of consensus, which might include a common terminology on interdisciplinary connections, with agreement on the meanings of micro, meso, macro, and global scales.

This investigation into the unification of the social sciences, while innovative in a sense, needs to be linked tightly to previous analysis, in order to avoid losing track of previous advances. As a general comment on social- 
science disciplines, one can say that the past half century has seen the rise of many new sub-disciplines defined by function and scale, but not a great deal of new work on linking the sub-disciplines to each other nor on characterizing the overall character of each discipline. Nonetheless, advances in disciplinary theory, systems theory, and information science can surely be collated to facilitate advances in the documentation of our changing, global, social system.

We chose the issue of migration to illustrate some of the benefits and challenges associated with using our proposed strategy to guide the construction of a world-historical dataset. Our intent was to explore numerous dimensions of an important social phenomenon, through highlighting issues relating to scale, level, aggregation, and organization within human populations. The results of this exercise, in conjunction with the findings from our sideby-side comparison of demography, economics, sociology, and political science, revealed the variances likely to be encountered when parsing the differences among the dynamics of our two social universes. We anticipate that future applications of this approach - augmented by world-historical data collection - will yield a more comprehensive understanding of historical dynamics of the human condition.

At the outset, we set the objective of delineating a set of methodological standards with which to guide interdisciplinary inquiry in the social and natural sciences. There are many steps to go before such inquiry can be seen as well established; here are the initial points we offer to this end.

- Rely on the principles of systems analysis to explore the disciplines and their interactions

- Define disciplinary systems in terms of framework, variables, processes, transitions

- Within a discipline, conduct analysis across topics (input-output analysis in economics)

- Also within a discipline, define its scales, its levels of aggregation, the various local theories, and linkage of multiple levels within a discipline: micro-meso-macro, various levels of aggregation along four axes (topic, social scale, time, space);

- Identify interdisciplinary links at varying scales. To specify details of interdisciplinary linkage, focus initially on linkage of population data to other data

- In tracing variables linking disciplines, consider compound variables according to the details of their composition and dimensions (for instance, populations shift from numerator to denominator to both in calculation of income per capita, population density, and rate of migration).

- For additional insights into properties of disciplines and their interactions, explore narratives of the development of thought in each discipline

- Develop steps toward a unified vocabulary to discuss interdisciplinary issues

In sum, for construction of Big Data centering on social issues, the methodology of interdisciplinary analysis relies on both old and new techniques. Reaffirming the strengths of the old, interdisciplinary analysis builds fundamentally on the methods established within each discipline. Charting new and broader terrain, interdisciplinary analysis requires attention to every aspect of aggregation and disaggregation in theory and data: detailed attention to the levels, dimensions, and overall scales; and equally close attention to the definition of each variable and to possible ambivalence as terms are given varying meanings in different fields.

\section{Appendix 1. Glossary: Properties of Symbolic Systems}


Adaptive systems. Systems that change in structure and function in response to problems of disorganization. In successful adaptations, systems respond to noise from outside the system in ways that turn some of the noise into information.

Chaos. A chaotic system is an analytical or symbolic system for which its dynamic interactions are governed by precise and unchanging relations among elements, but in which overall change is highly sensitive to initial conditions. Over the long term, tiny adjustments in initial conditions can bring chaotic system change.

Closed system. A system which is entirely isolated and self-sufficient, with no materials or influences crossing its borders.

Complex systems. The notion of complex systems is intended to distinguish those systems that have linkages and interactions beyond those required for basic operation of a system. So far, it has not proved possible to identify precisely the boundary between simple and complex systems. But among the characteristics listed to identify complex systems are that they include non-linear relationships (as between input and output), fractals (irregular forms are scale dependent), recursive symmetries between scale levels, and are sensitive to initial conditions. Complex systems can also be identified in narrative terms, in that they include contextuality and reflexivity, expression of purposes and motives, and temporal sensitivity (Newell 2001). In addition, systems allow for reproduction, self-regulation, and adaptation, though it may be that these properties are not restricted to complex systems. The purpose of this listing of characteristics of systems has been to highlight terminology that may be useful in focusing on the characteristics of overlapping, analytical systems so as to provide assistance in the project of constructing a global, historical archive. As we will see, several of these terms are applied elsewhere in this discussion.

Dimension. While this term retains is common meaning of the spatial, temporal and other dimensions of any physical object or theoretical variable, for the purposes of analyzing social systems we have also defined it more specifically as a set of four axes or domains: time, space, population, and topic. The terms "level" and "scale" are connected to this terminology.

Domain. This generic term refers to the range of space, time, populations or topics encompassed and treated as a unit or sub-unit, either in social practice or in theory.

Emergent properties. We define these properties as properties of systems, and in terms of differentiated scale within systems. In general, the interaction among elements of a system at a given scale may give rise, in real time, to a pattern of interaction and behavior at a more aggregated scale. In an example below, we will see how Balinese rice farmers built water temples that turned out to facilitate periodic meetings among the farmers, giving them a region-wide discursive network that enabled them to give productive responses to changes in climate or regulation that threatened their rice fields (Lansing and Kremer 1993; see also Appendix 2). The new property at a relatively aggregated level, once it has arisen, becomes part of the system. This then is an example of adaptive behavior of a social system. Note that the term "emergent properties" is used to refer to properties of "real world" systems. A related but less-studied question is whether the social science disciplines have emergent properties. "Each sub-system and ... even each plane of a multi-dimensional system can have its own emergent properties as well.”(Newell 2001, 7; Flood and Carson 1993, 17-19)

One of the benefits of the notion of emergent properties for the study of human society is that it argues that such properties reflect not simply the agency of some founding figure but also the realization of a structural potential 
that has gradually become manifest. The rise of empires is a good case in point: the surviving narratives focus especially on the will power and charisma of the founder, but the notion of emergent properties makes one think of the underlying potential. A second good case in point is the crystallization of nationhood among virtually all the peoples of the world within a period of two centuries. While efforts to justify the recognition of certain nations created a hierarchy treating some nations as more valid than others, an overall global mechanism brought them into existence, everywhere, just the same.

Entropy. The notion that, in closed systems, nature tends the bring change from order to disorder. As a corollary, the creation of order in one part of a system brings about more disorder in other parts of a system. (In open systems, the creation of order in one system brings more disorder in adjoining systems.) This notion, first conceptualized in the study of thermodynamics, has now been extended to many fields of study.

Feedback. In this property of all systems, information about a phenomenon at one time influences the same phenomenon at a later time. "Information" can take many forms; "circularity of action" is another phrase.

Level. This term refers to a position along a dimension. Levels can be ranked for chronological time and for the extent of time. Levels can be ranked for the extent of space, population, and topics, but any further ranking of levels along these dimensions requires additional criteria for hierarchy.

Narrative. The particular narrative to which we refer is that of the development of theory and research within each discipline: it records the advances but also the debates and dilemmas in analysis.

Open system. A system with open or porous borders. Its characteristics include input (materials and influences entering the system from outside) and output (materials and influences expelled from the system.

Scale. This term, parallel to "level," is taken as a more generic reference to the scope of social experience included in a given statement. "Large scale" refers to large regions, periods of time, or range of topics. But for lack of criteria ranking the dimensions in any order, it is hard to know whether large regions and short periods of time represent a larger scale than small regions and long periods of time. Nonetheless, "scale" is useful as a general descriptor of the scope of a system or sub-system.

Thresholds. For change within a system, a threshold is a transition point between alternate states (Liu, et al., 2007).

Teleology. Direction, purpose, and objectives in system behavior.

\section{Appendix 2: Emergent Properties}

Emergent properties are the characteristics or patterns of behavior that develop as a result of the collective activity of individual agents and entities, or a combination of the two. In turn, such properties may give rise to greater systemic complexity, which is measurable by a number of different parameters: the number of components or variables contributing to systemic operation, for instance, the nature of the system's output, the amount of entropy it possesses, its mode of propagation and evolution, or its subsequent interaction with the environment. If we treat populations as systems, and analyze them through the lenses of both the natural and social sciences, it is possible to trace the emergence of several unique properties. 
From the field of systems biology, for example, let us consider schooling, an aggregative phenomenon observed among many species, notably fish. More specifically, schooling is a display of harmonious, coordinated movement within a group of individuals that facilitates the exchange of information; such activity is thought to increase the evolutionary fitness of both the group and its constituents. Schools typically self-organize into highly structured patterns of shape and movement that enable the group to evade predation, a feat accomplished by individuals matching their behavior to that of their neighbors (Parrish et al. 2002). Because only groups of fish can form schools, and because schools themselves are complex phenomena resulting from simpler actions performed synchronously (i.e. collision avoidance and attraction to members of one's own species), school formation may be considered a population-level property emerging from the collective behavior of single fish. ${ }^{22}$

Although biological systems are replete with emergent phenomena, emergence is also a hallmark of social systems. Governments, clans, tribes, classes, and nations, for example, all illustrate social, economic, and political organization. Such organization allows for the emergence of culture and formalized social institutions (e.g. marriage, religion, and education). Although organization itself is not an emergent property of complex systems, the changes in systemic behavior resulting from the nonrandom arrangement of systemic components does facilitate the emergence of unforeseen properties. Let us consider the example of Balinese water temple networks. ${ }^{23}$ Over the centuries, Balinese farmers seeking to nourish their land have fashioned an extensive network of irrigation canals, punctuated by temples and shrines dedicated to Balinese deities. In addition to serving a religious function, these temples also act as rendezvous point for the farmers in a particular network (known as a subak) who must coordinate planting and irrigation strategies. A study of these farming networks shows that even in the presence of a disturbance in the overall system - uncoordinated planting, insect infestations, and low rain, for example - the subaks spontaneously reorganize to continue producing crops in the most optimal manner possible. Resilience, therefore - the ability to successfully recover from systemic disorder - is an emergent property of Balinese farming networks. While resilience does increase the complexity of the farming networks, it also contributes to systemic endurance and, more importantly, allows the system to adapt to environmental changes.

Some emergent properties are exclusive to the natural world while others materialize in a social context. Yet many arguably span both domains: the emergence of language in human populations is one such instance. Language expert James R. Hurford posits that linguistic evolution is largely attributable to the biological features that enable humans to produce speech sounds (phonetics), organize those sounds into sequences (syntax), form concepts and ideas (semantics), link sounds to said concepts (symbolic capacity), and engage in social interaction (pragmatics) (Christiansen and Kirby 2003). In addition to these, psychologist Steven Pinker also presents a major sociological factor responsible for the emergence of language: the need for a means of gathering and sharing information. In that vein, Pinker maintains that language fulfills a major role necessitated by "a humanly distinct cognitive niche. ${ }^{24}$ Given the sheer diversity associated with modern languages, and the ways in which this emergent property contributes to culture, communication, and identity, it is clear that language greatly amplifies the complexity of human populations.

It is crucial to note that the emergent properties in the aforementioned examples - schooling, resilience, and language - share a synergistic quality with respect to the constituents of the populations in question. In other words, these properties contribute to the overall function and dynamic of a population (the system) in such a way that it is difficult to reduce the resultant systemic behavior to the simple sum of phenomena at the micro-level: the movement of individual fish, the planting strategies of individual farmers, and the social and genetic changes enabling speech. Thus, because the emergence of these newer properties are not a direct function of existing systemic features, it might be said that emergent and prevailing properties share a nonlinear relationship (Newell 2001). 
From a teleological perspective, it appears that the function of emergent properties is simply to compound the intricacy of an already complex system. As exemplified by the Balinese water temple system, though, such properties may in fact contribute to systemic endurance and adaptation in a changing landscape. Similarly, language offers members of human populations the ability to engage in more sophisticated modes of communication in an increasingly complex social environment. In the context of interdisciplinary studies, however, emergent properties serve to elucidate the relationships between various scales and levels of analysis. From a procedural perspective, the study of emergent properties makes apparent the need for analytical tools specific various levels of aggregation within a population. Tracing emergent properties from multiple disciplinary domains also reveals the need to recognize the unique contributions of multi-level phenomena to the overall complexity of a system, and underscores the need for methodological tools sensitive enough to analyze these systemic features.

\section{NOTES}

${ }^{1}$ For the fullest formal statements on world history, see Bentley (2011) and Manning (2003).

${ }^{2}$ Chase-Dunn and Hall (1997). In practice, while we are happy to use "unifying the social sciences" as a slogan, our objective is much more narrow and specific. It is to use the factor of population and demographic variables to trace threads through the social-science disciplines.

${ }^{3}$ The overall strategy of building a world-historical data resource requires working from both the specific and general poles. This part of that work focuses on the bottom-up aspect of the overall task.

${ }^{4}$ Huxley (1946); Maurel (2010).

${ }^{5}$ The work of Darwin, while in the arena of natural science, must be considered a triumph in his linkage of the various scales from that of individual organisms to the level of whole species.

${ }^{6}$ One important exception was in the rise of multidisciplinary area studies programs, which encouraged study of Latin America, Asia, the Middle East, Russia and Eastern Europe, and Africa through disciplines including history, political science, anthropology, sociology, economics, and literature.

${ }^{7}$ Since the days of Norbert Wiener and Ludwig von Bertalanffy, researchers have been content to refer to those dynamics in terms built around the term "system" (Wiener 1950, von Bertalanffy 1968). In earlier usage, the term "solar system" goes back to the end of the seventeenth century.

${ }^{8}$ In a different formulation, Tsoukas and Hatch (2001), in describing complex systems, use the term "firstorder complexity" to refer to complexity in the world and use "second-order complexity" to refer to complexity in the accompanying theory.

${ }^{9}$ For instance, the term "large-scale analysis" could refer to an analysis encompassing a continent during a given year, a national unit over the course of two centuries, or the globe over the course of a century. 
${ }^{10}$ Faist (1997).

${ }^{11}$ For the case of migration theory, a notion has been developed that the universe of migration includes a grand grid of possible relationships which are gradually being addressed with theories on successive segments (Manning and Trimmer 2012).

${ }^{12}$ For an arresting display of multiple and overlapping scales, see "The Scale of the Universe 2", http://htwins.net.

${ }^{13}$ These two points can be confirmed with a quick historical scan of social-science theory. In seventeenth- and eighteenth-century European work, Hobbes, Locke, Rousseau, Malthus, etc., ranged across scales from the societal to the individual. In the nineteenth century Hegel and Marx sought to think in terms of humanity overall, while Marshall and Durkheim turned to an effort to work from an individual level outward.

${ }^{14}$ In a biological example, one may point to the subordination and repurposing of mitochondria within multicellular animals.

15 "To confront the passages between disciplines is to confront what Atlan calls complexity, large quantities of organizational information that appear to an observer as uncoded variety"(Paulson 1991, 45). "Complexity" is thus best seen as a relative rather than an absolute term within systems analysis. For an alternative approach, see Newell (2001). The notion of second-order complexity, as explicated by Tsoukas and Hatch (2001) and Casti (1986), implies that the posited complexity of a system is largely contingent upon the individual analyzing it.

${ }^{16}$ Paulson (1991), Newell (2001), and Tsoukas and Hatch (2001) each agree that narratives of theoretical change serve as devices for addressing complexities that are not yet rendered orderly by theory.

${ }^{17}$ Thacker (1990) strongly supports meta-analysis as an effective strategy for integrating scientific data in an age of rapid discovery and change. He does, however, suggest that meta-analytical strategies require further empirical testing.

${ }_{18}$ Additional disciplines that should be described according to a similar framework include anthropology, history, human geography, and psychology, along with the adjoining natural-science fields of health and climate.

${ }^{19}$ This condensed summation of sociology cannot encompass all the dimensions of an extensive and variegated discipline. For instance, it ranges from sociology of science to ethnomethodology and world-systems, including scholars with different orientations to data.

${ }^{20}$ As with sociology, the field of political science includes subfields ranging well beyond the approaches that are emphasized here.

${ }^{21}$ For the case of migration theory, a notion has been developed that the universe of migration includes a grand grid of possible relationships which are gradually being addressed with theories on successive segments (Manning and Trimmer 2012).

${ }^{22}$ As described in Olson (2008).

${ }^{23}$ As described in Lansing and Kremer (1993). 
${ }^{24}$ As quoted in Christiansen and Kirby (2003).

\section{REFERENCES}

Backhouse, Roger E., and Philippe Fontaine, eds. 2010. The History of the Social Sciences since 1945. Cambridge: Cambridge University Press.

Bentley, Jerry H. ed. 2011. Oxford Handbook in World History. Oxford: Oxford University Press.

Casti, J. 1986. On system complexity: Identification, measurement, and management. In J. Casti and A. Karlqvist, eds., Complexity, language, and life: Mathematical approaches (Berlin: Springer-Verlag).

Chase-Dunn, Christopher, and Thomas D. Hall. 1997. Rise and Demise: Comparing world Systems. Boulder: Westview Press.

Christiansen, Morten H., and Simon Kirby. 2003. Language Evolution. Oxford: Oxford University Press.

Faist, Thomas. 1997. "The Crucial Meso-Level.” Tomas Hammer, Grete Brochmann, Kristof Tamas, and Thomas Faist, eds., International Migration, Immobility and Development: Multidisciplinary Perspectives (Oxford: Berg Publishers), $187-218$.

Flood, R., and E. Carson. 1993. Dealing with Complexity: An Introduction to the Theory and Application of Systems Science, $2^{\text {nd }}$ ed. New York: Plenum Press.

Gerring, John. 2012. Social Science Methodology: A Unified Framework, $2^{\text {nd }}$ ed. Cambridge: Cambridge University Press.

Gibson, Clark C., Elinor Ostrom, and T. K. Ahn. 2000. "The concept of scale and the human dimensions of global change: a survey." Ecological Economics 32: 217 - 239.

Harvey, David L., and Michael Reed. 1996. "Social Science as the Study of Complex Systems," in L. Douglas Kiel and Euel Elliott, eds., Chaos Theory in the Social Sciences (Ann Arbor: University of Michigan Press).

Hayes, Nicky. 2000. "Doing Psychological Research: Gathering and Analysing Data.” Buckingham, UK: Open University Press.

King, Gary, Robert O. Keohane, Sidney Verba. 1994. Designing Social Inquiry: scientific inference in qualitative research . Princeton: Princeton University Press.

Lansing, J. Stephen, and James N. Kremer. 1993. "Emergent Properties of Balinese Water Temple Networks: Coadaptation on a Rugged Fitness Landscape." American Anthropologist 95: 97-114.

Liu, Jianguo, et al. 2007. "Complexity of Coupled Human and Natural Systems." Science 317: 1513-1516; doi 10.1126.

Lovelock, James. 2009. The Vanishing Face of Gaia. New York: Basic Books.

Manicas, Peter T. 2006. A Realist Philosophy of Social Science: Explanation and Understanding. Cambridge: Cambridge University Press.

Manning, Patrick. 2003. Navigating World History: Historians Create a Global Past. New York: Palgrave Macmillan.

Manning, Patrick, with Tiffany Trimmer. 2012. Migration in World History, $2^{\text {nd }}$ ed. London: Routledge.

Maurel, Chloé. 2010. Histoire de l'UNESCO: Les trente premières années. 1945 - 1974. Paris: L'Harmattan.

Meyer, William B. and B.L. Turner II. 1992. "Human Population Growth and Global Land-Use/Cover Change." Annual Review of Ecology and Systematics 23: 39-61.

Newell, William H. 2001. “A Theory of Interdisciplinary Studies.” Issues in Integrative Studies 19: 1-25. 
Olson, Gustaf. 2008. “Emergent Schooling Behavior in Fish.” http://guava.physics.uiuc.edu/ 〜nigel/courses/569/Essays_Fall2008/files/olson.pdf.

Overton, W.F. 2006. "Developmental Psychology: Philosophy, Concepts, Methodology.” In R.M. Lerner (Ed.). Theoretical Models of Human Development (New York: Wiley), 18-88.

Parrish, J.K., S. V. Viseido, and D. Grunbaum. 2002. "Self-Organized Fish Schools: An Examination of Emergent Properties." The Biological Bulletin 202: 296-305.

Paulson, William. 1991. "Literature, Complexity, Interdisciplinarity." In N. Katherine Hayles, ed., Chaos and Order: Complex Dynamics in Literature and Science (Chicago: University of Chicago Press), 37 - 53.

Robinson, W.S. 1950. "Ecological Correlations and the Behavior of Individuals." American Sociological Review 15: 351-357. doi: $10.2307 / 2087176$.

Scoones, I. 1999. "New Ecology and the Social Sciences: Prospects for a Fruitful Engagement?" Annual Review of Anthropology 28: 479-507.

Thacker, Stephen B. 1990. "Meta-Analysis: A Quantitative Approach to Research Integration.” Annetine C. Gelijns, ed.,, Modern Methods of Clinical Investigation (Washington, DC, National Academy Press), vol. 1: 88-97.

Tsoukas, Haridimos and Mary Jo Hatch. 2001. "Complex thinking, complex practice: The case for a narrative approach to organizational complexity." Human Relations 54: 979-1013.

Van Strien, Pieter J. 1997. "Toward a Methodology of Psychological Practice: The Regulative Cycle.” Theory and Psychology 7: 683 - 700.

Von Bertalanffy, Ludwig. 1968. General System Theory: Foundations, Development, and Applications, rev. ed. New York: Braziller.

Wiener, Norbert. 1950. The Human Use of Human Beings: Cybernetics and Society. Boston: Houghton Mifflin. Wilson, Edward O. 1999. Consilience: The Unity of Knowledge. New York: Vintage Books.

(cc) EY Articles in this journal are licensed under a Creative Commons Attribution 3.0 United States License.

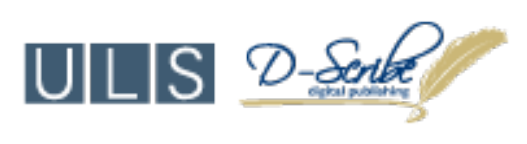

This journal is published by the University Library System of the University of Pittsburgh as part of its D-Scribe Digital Publishing Program and is cosponsored by the University of Pittsburgh Press. 